\title{
Correction to: Future projection of cancer patients with cardiovascular disease in Japan by the year 2039: a pilot study
}

\author{
Yuji Okura' ${ }^{1}$ - Tsugumi Takayama ${ }^{2} \cdot$ Kazuyuki Ozaki $^{2} \cdot$ Hiroshi Tanaka $^{3} \cdot$ Akira Kikuchi $^{4} \cdot$ Toshihiro Saito $^{5}$. \\ Toshiki Tanigawa $^{5} \cdot$ Yasumasa Takii $^{6} \cdot$ Hiroshi Seki $^{7} \cdot$ Tatsuya Takenouchi $^{8} \cdot$ Takaaki Chou $^{9} \cdot$ Nobuaki Sato $^{10}$. \\ Naohito Tanabe ${ }^{11} \cdot$ Tohru Minamino ${ }^{2}$
}

Published online: 22 April 2019

(c) Japan Society of Clinical Oncology 2019

\section{Correction to: International Journal of Clinical Oncology https://doi.org/10.1007/s10147-019-01426-w}

In the original publication, in a title of Table 2, the sentence that reads as, "Growth rate of cancer, cancer without CVD and cancer with CVD between 2015-2010" should read as, "Growth rate of cancer, cancer without CVD and cancer with CVD between 2015-2019”.

The original article can be found online at https://doi.org/10.1007/ s10147-019-01426-w.

Yuji Okura

okuray@niigata-cc.jp

1 Department of Onco-cardiology, Niigata Cancer Center Hospital, Chu-o-ku Kawagishicho 2-15-3, Niigata 951-8560, Japan

2 Department of Cardiovascular Biology and Medicine, Niigata University Graduate School of Medical and Dental Sciences, Niigata, Japan

3 Department of Respiratory Medicine, Niigata Cancer Center Hospital, Niigata, Japan

4 Department of Gynecology, Niigata Cancer Center Hospital, Niigata, Japan

5 Department of Urology, Niigata Cancer Center Hospital, Niigata, Japan
6 Department of Gastroenterological Surgery, Niigata Cancer Center Hospital, Niigata, Japan

7 Department of Diagnostic Radiology, Niigata Cancer Center Hospital, Niigata, Japan

8 Department of Dermatology, Niigata Cancer Center Hospital, Niigata, Japan

9 Department of Hematology, Niigata Cancer Center Hospital, Niigata, Japan

10 Department of Breast Oncology, Niigata Cancer Center Hospital, Niigata, Japan

11 Department of Health and Nutrition, Faculty of Human Life Studies, University of Niigata Prefecture, Niigata, Japan 